\title{
Epidural Pneumatic Pseudocyst with Lumbar Radiculopathy: Two Case Reports and Review of the Literature
}

\author{
Bong Hyun Jin, Jong Un Lee, Tae-Hun Kim, Ki-Hong Kim, Man Kyu Choi, Dae-Hyun Kim \\ Department of Neurosurgery, Daegu Catholic University Medical Center, Catholic University of Daegu College of Medicine, Korea
}

Corresponding Author:

Dae-Hyun Kim, MD, PhD

Department of Neurosurgery, Daegu Catholic

University Medical Center, Catholic University of

Daegu College of Medicine, 33, Duryugongwon-ro

17-gil, Nam-gu, Daegu 42472, Korea

Tel: $+82-53-650-3495$

Fax: +82-53-650-4932

E-mail: daehkim@cu.ac.kr

Received: May 15, 2019

Revised: June 3, 2019

Accepted: June 7, 2019
Intradiscal vacuum phenomenon is commonly shown in radiographic studies of the degenerative spine. In terms of pathophysiology, intradiscal gas migrates to the epidural space, and then it could compress neural tissues and lead to severe radicular pain and/or neurologic deficits. Herein, we report two patients with lumbar radiculopathy resulting from gas-containing pseudocysts at the L5-S1 space, including our treatment and literature review. One patient had been underwent L5S1 partial laminectomy and removal of the gas-containing pseudocyst, and the other underwent right selective L5 root block. After the procedures, they didn't have any remarkable complaints. In the present study, we suggest that surgical decompression may be effective in patients who were untreatable with conservative treatment.

Key Words: Intradiscal vacuum phenomenon, Epidural space, Intervertebral disc degeneration, Gas pseudocyst, Radiculopathy

\section{INTRODUCTION}

Intradiscal vacuum phenomenon represents collection of gas into the intervertebral disc space. It is a relatively common radiologic finding ${ }^{5}$. Although the clinical significance of intradiscal gas is not significant, gas in the spinal canal is meaningful in terms of neural compression. The gas formation in intervertebral disc space may occur as a result of disc degeneration and migration of gas in the intervertebral space to the spinal canal could lead to spinal cord and/or root compression, triggering radiating pain and neurological symptoms ${ }^{9}$.

However, gas-containing pseudocysts causing lumbar radiculopathy are relatively rare ${ }^{10)}$, and a few cases have been reported. Sometimes, epidural gas-containing cystic lesion may be misdiagnosed as epidural mass or disc herniation on magnetic resonance imaging (MRI), because patient's symptoms are nonspecific and cannot be distinguishable from those of degenerative disease.

We report two patients with radiculopathy resulting from gas-containing pseudocysts on the L5-S1 space. In addition, we report the diagnostic tool and treatment options of symptomatic epidural gas-containing pseudocyst based on our cases with review of previous studies.

\section{CASES REPORT}

\section{Case 1}

A 74-year-old female patient with a 5-month history of right leg radiating pain on posterior aspect admitted to our clinic. She had difficulty in lying and walking due to the severe leg pain. She had presented poor response after 5 months of conservative treatment at other hospital. The pain and sensory deficit were localized to right S1 sensory dermatome. VAS (visual analogue scale) score for leg pain was 8 points. Physical examination revealed the weakness of plantar flexion of right great toe (Medical Research Council power Grade 4/5). Electromyography and nerve conduction study showed the radiculopathy of right S1 dermatome.

On MRI, the lesion was located on ventral side of L5-S1 spinal canal (subarticular area). It was oval shape with homogenous hypo-intensity on T1- and T2-weighted images. Computed tomography (CT) scan also revealed intradiscal vacuum phenomenon on L4-5 and L5-S1 levels. The lesion was hypodense and could be diagnosed as an epidural gas-containing lesion (Fig. 1). It had been found at the same area on CT scan that has been performed 21 months ago. There was no change 

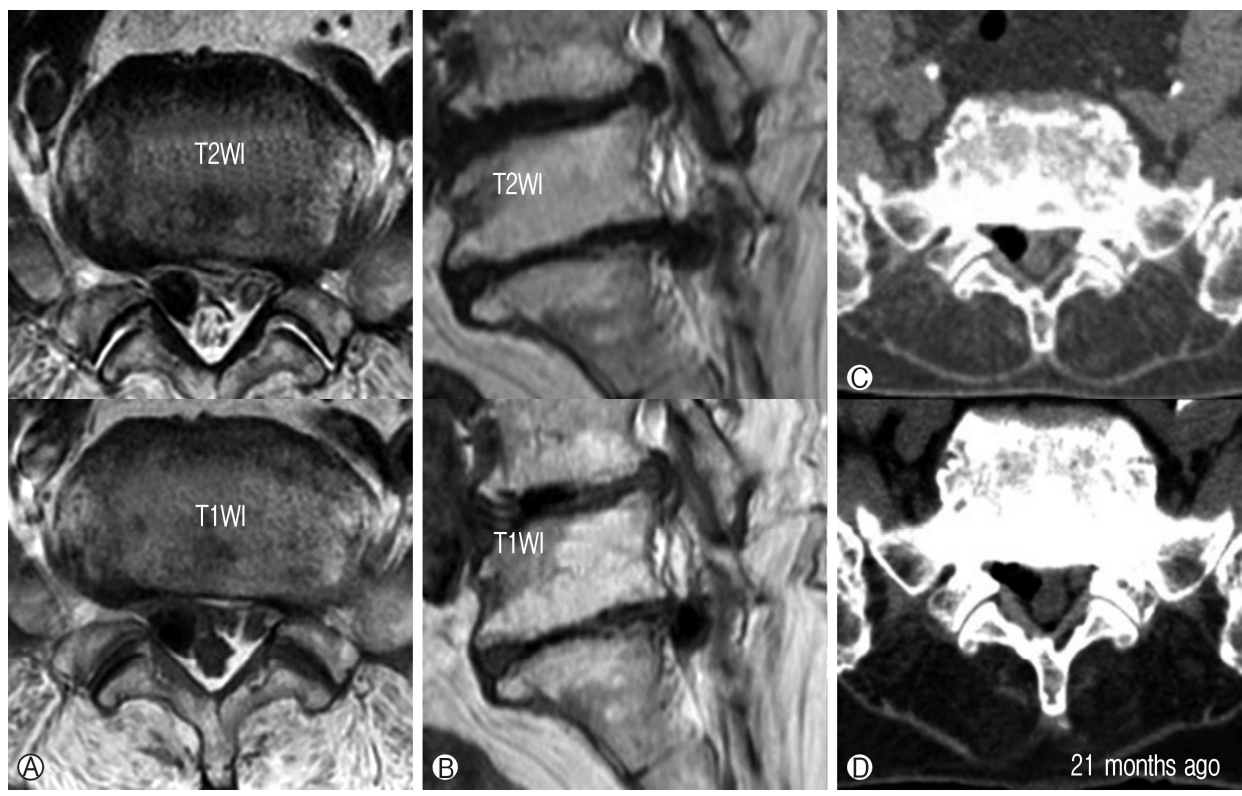

Fig. 1. 74-year-old female patient with a 5-month history of right leg radiating pain. Axial (A) and sagittal (B) views on T1- and T2-weighted images demonstrate a hypo-intense lesion on MRI. Axial view (C) of CT scan showing a hypodense lesion within the spinal canal at the L5-S1 level. Axial (D) view on CT scan that had been examined 21 months ago.

of size during two years. We performed L5-S1 partial laminectomy and removal of epidural gas-containing lesion using the minimal invasive tubular retractor. During the operation, gas-containing cyst lesion, which was compressing dural sac, was detected on ventral aspect of spinal canal. When we punctured the thin wall of the gas-containing lesion using micro-scissors, foam was observed abruptly after gas leakage. The lesion was excised and completely removed. In histopathological examination, thin cartilaginous membrane surrounding the gas was identified as a pseudocyst (Fig. 2). After the surgery, leg pain was improved, dramatically (VAS; 8 to 1$).$

\section{Case 2}

A 69-year-old female patient with a 3-month history of right leg radiating pain was admitted to our medical center. The pain and sensory deficit were localized to right L5 sensory dermatome. Dorsiflexion strength of right great toe was slightly decreased (Grade 4+/5). Twenty months ago, she was underwent decompressive laminectomy for severe stenosis at L3-4 and L4-5 level in other hospital.

On MRI, oval shaped lesions were located on both intervertebral foramina at L5-S1 level. It was homogenous hypo-intensity on T1- and T2-weighted images. CT scan revealed vacuum phenomenon, the right one was larger than the left. The lesion was hypodense and could be diagnosed as an epidural gas-containing lesion (Fig.3). The lesion was found at the same site in previous CT scan (performed twenty month ago). We performed the right $L 5$ nerve root block and then symptoms were improved.
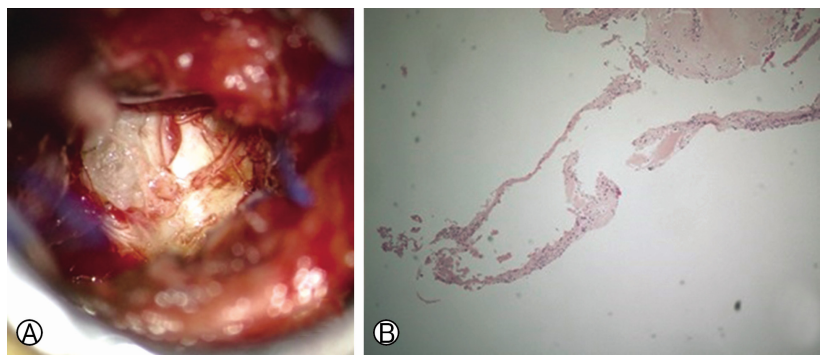

Fig. 2. Surgical findings showing gas-containing cyst lesion and foam, observing after gas-leakage (A). Cartilaginous thin membrane surrounding the gas was identified as the pseudocyst (B), enlarged, ×200) in histopathologic sample.

\section{DISCUSSION}

Gas collections have been described in literatures regardless of spinal segment, including the disc space, vertebral structure, epidural and intradural spaces, synovial cysts and facet joints ${ }^{5}$. Intradiscal vacuum phenomenon is commonly detected in radiologic studies of the degenerative spine. Previously reported cases had degenerative disc disease and vacuum phenomenon at lesioned level ${ }^{9)}$. The pathogenesis of gas-containing pseudocyst may be explained by the herniation of intradiscal gas together with vacuum phenomenon. Our cases may be characterized by discal cyst formation after disc herniation with mass effect, then herniated disc might disappear and cyst might remain. It is hypothesized that there may be gas remai- 

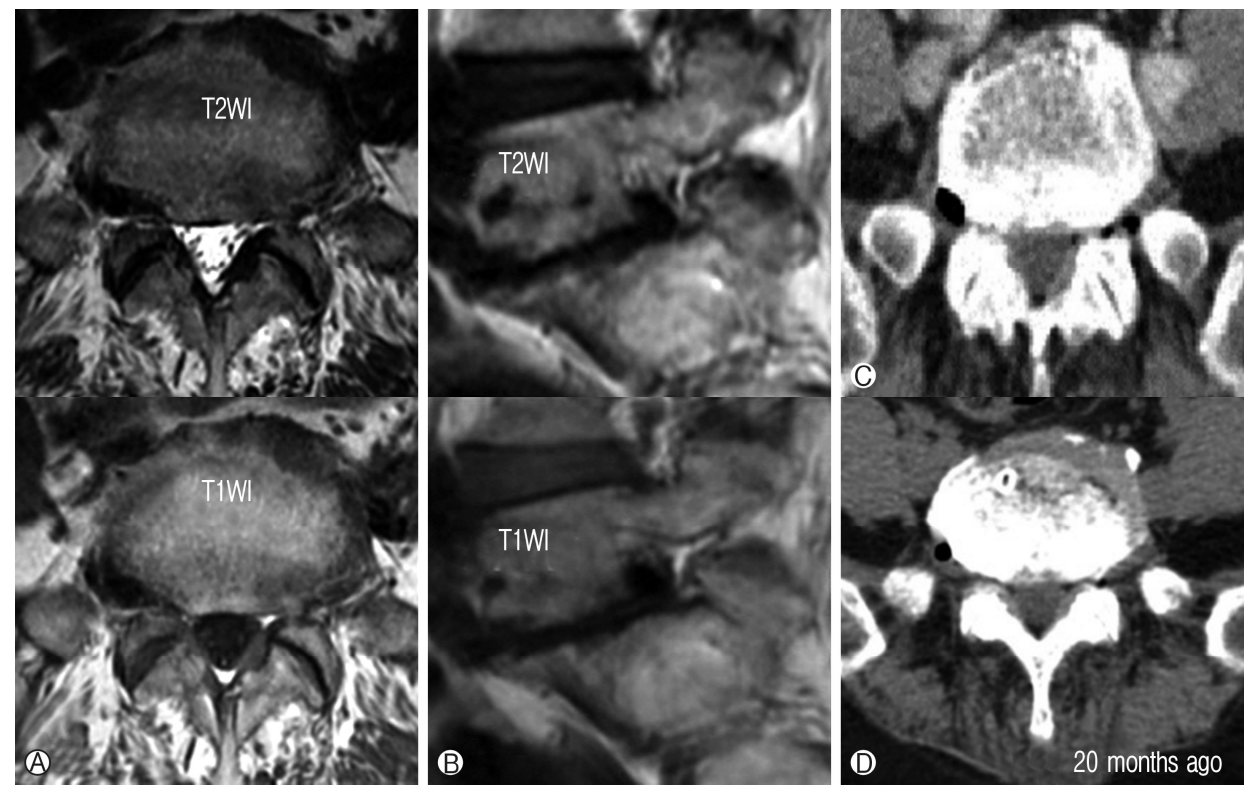

Fig. 3. 69-year-old female patient with a 3-month history of right leg radiating pain. Axial (A) and sagittal (B) views on T1- and T2-weighted images demonstrate a hypo-intense lesion on MRI. Axial view (C) of CT showing a hypodense lesion within the spinal canal at the L5-S1 level. Axial view (D) on CT scan that had been examined 20 months ago.

ning in the vacant area due to the fluid resolution in the cyst region. The histologic findings of our case with gas-containing pseudocyst revealed cystic walls composed of cartilaginous thin membrane. Thus, gas-containing pseudocyst may be related to degenerative change and intradiscal vacuum phenomenon.

Intradiscal gas might migrate to the epidural space, and herniated intraspinal gas could form the lesion" ${ }^{9}$. Kakitsubata et al. $^{8)}$ had reported four cases in which they use CT myelography and discography as diagnostic tool. They detected a relation between the gas-containing pseudocyst and intervertebral disc on CT discography visualized as contrast material flow. The gas was distributed in an area from the central spinal canal to the extraforaminal area. The location of lesion was appeared in various areas of the lumbar spine. We classified the lesion into locations of level and compression type in previous studies including our cases ${ }^{1-4,6-15)}$. The compression to neural tissues type was divided into three types: canal (central to paracentral), foraminal and extraforaminal. Twenty eight were in the canal $(57.1 \%)$, thirteen in the foramen $(26.5 \%)$, and eight were extraforaminal (16.3\%). We could confirm that pseudocyst was commonly found in L4-5 (44.9\%) and L5-S1 (34.7\%) levels. Epidural gas-containing pseudocyst could compress neural tissue and lead to severe radicular pain or neurologic deficits. In several literatures, there were no diagnostic algorithms for gas-containing pseudocyst. The lesion may be misdiagnosed as epidural mass or herniated intervertebral discs on MRI. It is seen as homogenous hypo-intensity or a signal void on T1and T2-weighted images. On the other hand, the presence of gas within pseudocyst is well detected on CT scan. It is hypodense and its Hounsfield unit value is a negative similar to an air. Therefore, CT scan is essential for diagnosis of the epidural pneumatic pseudocyst ${ }^{9)}$.

Treatment options for epidural pneumatic pseudocyst are still not established. Anda et al. ${ }^{2}$ published a case of 40 -years-old patient with left leg radiating pain. They used CT scan for diagnosis and detected gas-containing pseudocyst on L5-S1 left extraforaminal space. After conservative therapy, symptom had been improved. We consider that small gas-containing pseudocysts spontaneously might be resolve without invasive procedure, and radicular pain could be improved after conservative treatment in patients with mild radicular pain and no neurologic deficits.

However, invasive procedures were required in cases that had severe pain with neurologic deficits, refractory to conservative therapies. Invasive treatments for gas-containing pseudocyst such as needle aspiration and operation had been reported $^{3)}$. Bosser et al. ${ }^{3)}$ reported a 60 -years old patient treated CT-guided needle aspiration. His symptoms transient improved, but five days later, he relapsed and underwent surgery.

\section{CONCLUSION}

In the present study, we suggest that lumbar gas-containing pseudocysts could induce radicular pain and/or symptoms of neurologic compromise. The gas-containing pseudocysts of two patients in our case were not spontaneously absorbed and caused radicular pain for a long time. Therefore, we suggest that surgical decompression may be effective in patients who were untreatable with conservative treatment. 


\section{Conflict of Interest}

The authors report no conflict of interest concerning the materials or methods used in this study or the findings specified in this manuscript.

\section{Informed Consent}

Written informed consent was obtained from all patients for publication of this manuscript and any accompanying images.

\section{REFERENCES}

1. Akhaddar A, Eljebbouri B, Naama O, Boucetta M: Sciatica due to lumbar intraspinal gas pseudocyst. Intern Med 49:2647, 2010

2. Anda S, Stovring J, Ro M: CT of extraforaminal disc herniation with associated vacuum phenomenon. Neuroradiology 30:7677, 1988

3. Bosser V, Dietemann JL, Warter JM, Granel de Solignac M, Beaujeux R, Buchheit F: L5 radicular pain related to lumbar extradural gas-containing pseudocyst. Role of CT-guided aspiration. Neuroradiology 31:552-553, 1990

4. Cheng TM, Link MJ, Onofrio BM: Pneumatic nerve root compression: epidural gas in association with lateral disc herniation. Report of two cases. J Neurosurg 81:453-458, 1994

5. Coulier B: The spectrum of vacuum phenomenon and gas in spine. JBR-BTR 87:9-16, 2004

6. Kaiser MC, Capesius P, Veiga-Pires JA, Bruch JM: Recognition of gas-containing disc herniation on lateral CT-scoutview. Neuroradiology 29:98, 1987

7. Kakitsubata Y, Theodorou SJ, Theodorou DJ, Yuko M, Ito Y, Yuki Y, et al: Symptomatic epidural gas cyst associated with discal vacuum phenomenon. Spine (Phila Pa 1976) 34:E784-789, 2009

8. Kawaguchi S, Yamashita T, Ida K, Ikeda T, Ohwada O: Gas-filled intradural cyst of the lumbar spine. Case report. J Neurosurg 95:257-259, 2001

9. Kuh SU, Heo DH, Kim KS, Cho YJ: Lumbar epidural gas-containing pseudocysts as a cause of severe radicular pain. Joint Bone Spine 78:398-401, 2011

10. Lee DY, Lee SH: L2 radicular compression caused by a foraminal extradural gas pseudocyst. J Korean Neurosurg Soc 47:232234, 2010

11. Pierpaolo L, Luciano M, Fabrizio P, Paolo M: Gas-containing lumbar disc herniation. A case report and review of the literature. Spine (Phila Pa 1976) 18:2533-2536, 1993

12. Ricca GF, Robertson JT, Hines RS: Nerve root compression by herniated intradiscal gas. Case report. J Neurosurg 72:282-284, 1990

13. Ryu KS, Rathi NK, Shin MH, Park CK: Gas-containing disc herniations: dual nerve root compression at a single disc level. Neurol Med Chir (Tokyo) 52:649-651, 2012

14. Yun SM, Suh BS, Park JS: Symptomatic epidural gas-containing cyst from intervertebral vacuum phenomenon. Korean J Spine 9:365-368, 2012

15. Zhu B, Jiang L, Liu XG: Transforaminal endoscopic decompression for a giant epidural gas-containing pseudocyst: A case report and literature review. Pain Physician 20:E445-E449, 2017 